\title{
Tyrosine Hydroxylase Immunoreactive Neurons Throughout the Hypothalamus Receive Glutamate Decarboxylase Immunoreactive Synapses: A Double Pre-embedding Immunocytochemical Study with Particulate Silver and HRP
}

\author{
Anthony N. van den Pol \\ Section of Neurosurgery, Yale University Medical School, New Haven, Connecticut 06510
}

Silver-intensified gold (SIG) particles were used for light- and electron-microscopic immunocytochemical localization of neuronal antigens, and the SIG method was compared with related heavy-metal methods for the purpose of dual ultrastructural localization of neurotransmitter-related antigens. SIG immunostaining was combined with peroxidase immunostaining to allow simultaneous study of differentially labeled tyrosine hydroxylase and glutamate decarboxylase immunoreactive neurons in the medial hypothalamus.

A number of electron-dense markers that might be of use in double immunostaining for light and electron microscopy were examined, either with a simple nitrocellulose dot-blot method or on Formvar-coated slot grids. Of these, silver-intensified 5 nm colloidal gold was the most effective. Silver intensification of colloidal silver and of peroxidase reaction product also showed promise for combined LM and EM double-immunolabeling studies. Since the silver-intensification procedure used here intensifies both gold and peroxidase, in experiments involving double staining, the silver-intensified gold procedure should be used for the first antigen and nonintensified HRP for the second.

Presumptive dopaminergic neurons containing the enzyme tyrosine hydroxylase were located throughout the hypothalamus with SIG immunostaining. In the same areas where frequent tyrosine hydroxylase immunoreactive neurons were found, many axons and bouton terminals were also found with antisera against GABA or against the GABA-synthesizing enzyme glutamate decarboxylase. Areas containing cells immunoreactive for tyrosine hydroxylase and stained with SIG and axons immunoreactive for glutamate decarboxylase and stained with peroxidase included the periventricular area (A14), the arcuate nucleus (A12), the dorsomedial hypothalamus/zona incerta area (A13), the posterior hypothalamus (A11), the medial paraventricular nucleus, and dorsal to the supraoptic nucleus, in addition to the preoptic area near the third ventricle and dorsally adjacent to the anterior commissure. For comparison, the SIG procedure was also used to stain dopaminergic neurons outside the hypothalamus in the substantia nigra and ventral tegmental area.

Double immunocytochemical staining of two different neurotransmitter-related antigens allowed examination with both light

\footnotetext{
Received Mar. 25, 1985; revised June 3, 1985; accepted June 4, 1985.

I thank Drs. W. Oertel, D. Schmechel, I. Kopin, and M. Tappaz for glutamate decarboxylase antiserum, Dr. P. Somogyi for GABA antiserum, Dr. J. Powell for tyrosine hydroxylase antiserum and suggestions, and G. Collins and K. Szigeti for histological assistance. Support provided by NIH NS 16296, NIH NS 10174, and the American Parkinson Disease Association.

Correspondence should be addressed to Dr. van den Pol, Section of Neurosurgery, Yale University Medical School, 333 Cedar St., New Haven, CT 06510.

Copyright (c) 1986 Society for Neuroscience 0270-6474/86/030877-15\$02.00/0
}

and electron microscopy. By virtue of a large silver shell formed around the colloidal gold particle and its adsorbed immunoglobulin or protein $A$, cross-reactivity of the first set of immunoreagents stained with particulate silver and a second set stained with peroxidase could be reduced or eliminated. To test its versatility, the SIG methodology was used to stain five other putative neurotransmitter-related antigens in the hypothalamus, including glutamate decarboxylase, somatostatin, prolactin, luteinizing hormone releasing hormone, and neurophysin in frozen sections or sections embedded in plastic, polyethylene glycol, or paraffin.

In all areas examined ultrastructurally, including the arcuate nucleus (A12), the periventricular area (A14), the medial paraventricular nucleus, and the dorsomedial hypothalamus, glutamate decarboxylase immunoreactive boutons stained with peroxidase were found in direct contact with tyrosine hydroxylase immunoreactive dendrites and perikarya stained with the silverintensified gold procedure. As glutamate decarboyxlase immunoreactive boutons were found in synaptic contact with tyrosine hydroxylase immunoreactive neurons throughout the hypothalamus, the inhibitory amino acid transmitter GABA may play a widespread role in modulating the electrical activity of dopamine neurons.

Cells containing immunoreactive tyrosine hydroxylase (TH), the rate-limiting enzyme in dopamine synthesis, are found throughout the hypothalamus. Previous work has suggested that the presence of $\mathrm{TH}$ in perikarya of the hypothalamus is indicative of a dopaminergic neuron. Dopamine beta-hydroxylase and phenylethanolamine- $N$-methyl transferase, the synthetic enzymes involved in norepinephrine and epinephrine synthesis, although found in axons within the hypothalamus, have not been found in medial hypothalamic somata or dendrites (Bjorklund et al., 1975; Fuxe, 1964; Hökfelt et al., 1974; Swanson and Hartman, 1975; Swanson et al., 1981; van den Pol et al., 1984). Dopamine neurons of the hypothalamus play an important role in regulation of pituitary tropins, particularly prolactin, and also are involved in many of the other functions of the hypothalamus (Brown et al., 1972; Lloyd et al., 1975; Lookingland and Moore, 1984; MacLeod and Lehmeyer, 1974; Tilders et al., 1979; Weiner and Ganong, 1978).

Axons that are immunoreactive for glutamate decarboxylase (GAD), the enzyme responsible for synthesis of the inhibitory amino acid neurotransmitter GABA (Wu, 1983), are found in many regions of the hypothalamus. The present study utilizes a recently developed double-staining method (van den Pol, $1984 \mathrm{~b}, 1985$ a) that allows examination of two differentially labeled antigens at both the light=microscopic and electron-microscopic level. The possible influence of GABA-containing 
Table 1. Protocol for double immunostaining with SIG and HRP

1. Fix ( $4 \%$ paraformaldehyde and $0.1 \%$ glutaraldehyde)

2. Sucrose infiltration (until block sinks)

3. Freeze block in liquid nitrogen, thaw, freeze again

4. Cut $30 \mu \mathrm{m}$ sections on Vibratome

5. TBS buffer ( $\mathrm{pH} 8.2$ ) with glycine, lysine, $1 \%$ BSA (1-15 hr)

6. First primary antiserum: rabbit anti-TH (overnight)

7. Wash in TBS $(6 \times 10 \mathrm{~min})$

8. TBS and $1 \%$ BSA ( $30 \mathrm{~min})$

9. Goat anti-rabbit IgG adsorbed to $5 \mathrm{~nm}$ gold for $2 \mathrm{hr}$ (or protein A-adsorbed to $5 \mathrm{~nm}$ gold for $2 \mathrm{hr}$ )

10. TBS buffer $(5 \times 10 \mathrm{~min})$

11. Rabbit anti-goat FITC (use only on a few sections to ensure specific gold label is in tissue)

12. Citrate buffer, or distilled water $(3 \times 5 \mathrm{~min})$

13. Intensify with silver solution $(3 \mathrm{~min}$ to $2 \mathrm{hr}$ )

14. Citrate buffer $(3 \times 5 \mathrm{~min})$

15. TBS $(3 \times 5 \mathrm{~min})$

16. $5 \%$ Normal rabbit serum in TBS $(1 \mathrm{hr})$

17. Second primary antiserum: sheep anti-rat GAD (overnight)

18. TBS $(6 \times 10 \mathrm{~min})$

19. Biotin rabbit anti-sheep $\mathrm{IgG}(45 \mathrm{~min})$

20. TBS $(4 \times 10 \mathrm{~min})$

21. Avidin-biotin-peroxidase complex $(45 \mathrm{~min})$

22. TBS $(4 \times 5 \mathrm{~min})$

23. Tris ( $\mathrm{pH} 7.6)$

24. $\mathrm{DAB}$ and hydrogen peroxide

25. Tris $(3 \times 10 \mathrm{~min})$

26. Phosphate buffer $(2 \times 10 \mathrm{~min})$

27. $1 \%$ Osmium tetroxide in phosphate buffer $(1 \mathrm{hr})$

28. Dehydrate in ethanol and propylene oxide

29. Flat embed in Epon on glass slides pretreated with liquid release agent

30. Select area of interest with LM, photograph, remove from slide

31. Re-embed in flat portion of Beem capsule

32. Cut ultrathin sections

33. Stain with lead citrate and uranyl acetate if required (while the SIG deposit is easy to see on contrasted sections, peroxidase is not; therefore, it is best to examine some sections without counterstaining)

boutons on dopamine-containing cells in the medial hypothalamus was studied by examining synaptic interaction between neurons containing the synthetic enzymes for dopamine or GABA.

Double immunostaining at the ultrastructural level with two different electron-dense markers allows the determination of the neurotransmitter indentity of both the pre- and postsynaptic neurons. The ability to identify neurons on the basis of their

Figure 1. Silver intensification dot blots. A, Colloidal gold of 5, 10, 20 , and $40 \mathrm{~nm}$ were spotted on nitrocellulose and intensified with the silver. Even at dilutions of colloidal gold at 1:20,000, a faint staining could be detected. Prior to intensification, only the first dilution, 1:20, was visible as a pale pink spot. $B$, The same silver-intensification procedure can also be used to intensify protargol $(\mathrm{Pr})$. $C$, Silver bound to protein by a thiolactone procedure $(\mathrm{Ag}-\mathrm{T})$ was slightly visible after silver intensification, but even in concentrated form was less visible than SIG. $D-F$, Silver intensification at the level of individual gold particles: 5 $\mathrm{nm}$ colloidal gold $(D), 5 \mathrm{~nm}$ gold intensified for $30 \mathrm{sec}(E)$, and $120 \mathrm{sec}$ with constant agitation $(F)$. Bar, $180 \mathrm{~nm}$.

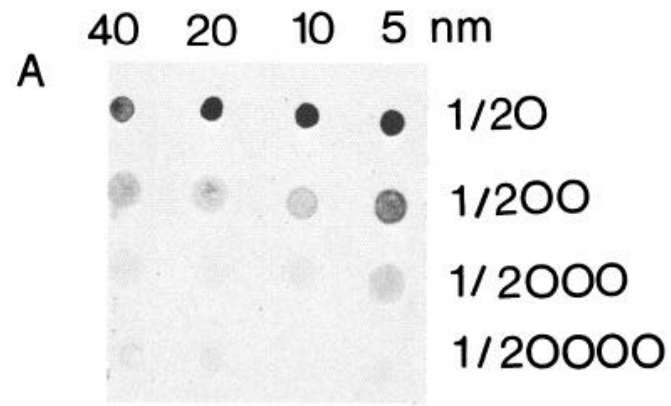

B

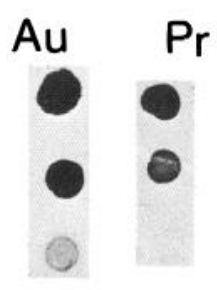

C Au Ag-T

D

$5 \mathrm{~nm} \mathrm{Au}$.

$E$

$30 \mathrm{sec}$

$120 \mathrm{sec}$

$\mathbf{F}$

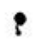

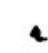



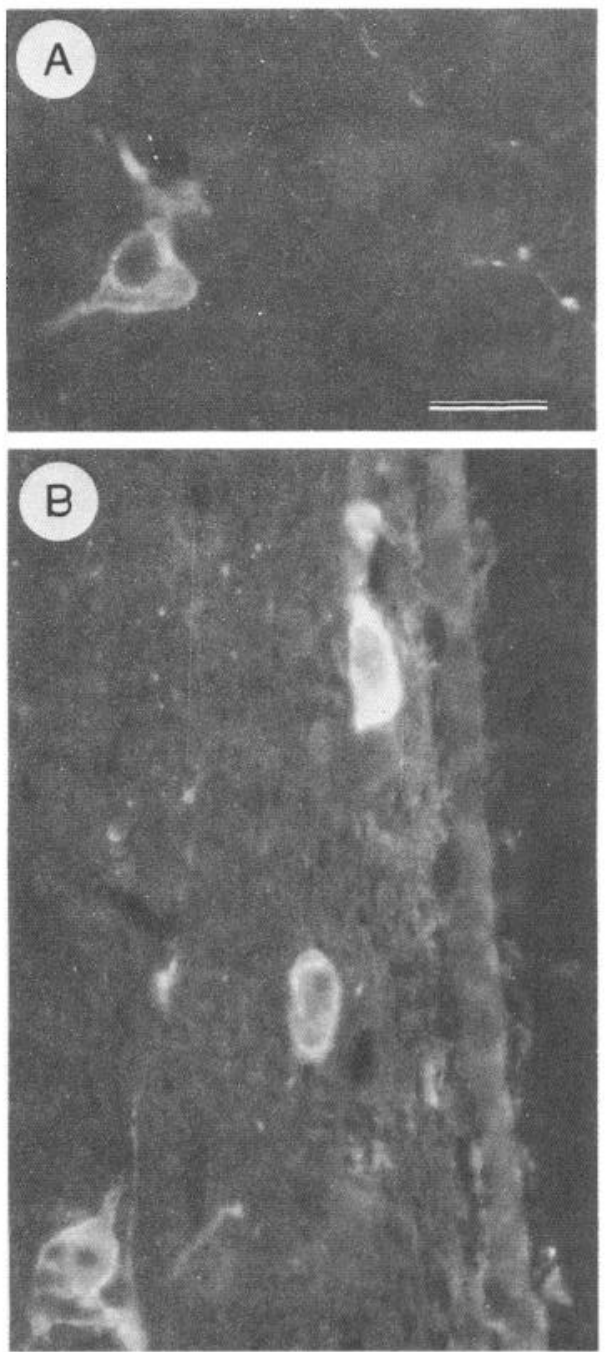

Figure 2. $A$ and $B$, After immunostaining with a primary antisera against TH of neurophysin (van den Pol, 1984), goat anti-rabbit IgG adsorbed to $10 \mathrm{~nm}$ gold was used to label the primary antisera. Since the gold staining was not visible with light microscopy, rabbit anti-goat IgG coupled to FITC was used to localize the neurons containing gold. Bar, $18 \mu \mathrm{m}$.

putative transmitter is particularly important in an area such as the medial hypothalamus, where most neurons may otherwise be indistinguishable. In addition to having two different immunocytochemical markers which can be differentiated with the EM, the procedures allow localization of each of the markers with light microscopy.

In the present paper, a number of different electron-dense markers are compared. Intensification of the size, electron density, and light visibility of many of these markers was attempted using deposition of silver at the site of the preexisting heavy metal. Based on the differential localization of their respective synthetic enzymes, the present paper examines GABAergic innervation of dopamine neurons throughout the medial hypothalamus and preoptic area, concentrating on the arcuate and paraventricular nuclei and the periventricular area.

Figure 3. GAD immunoreactivity: HRP. Large numbers of axons and boutons immunoreactive for GAD and stained with peroxidase are found in $A$, the arcuate nucleus $(A R C)$, in $B$, the hypothalamic peri-
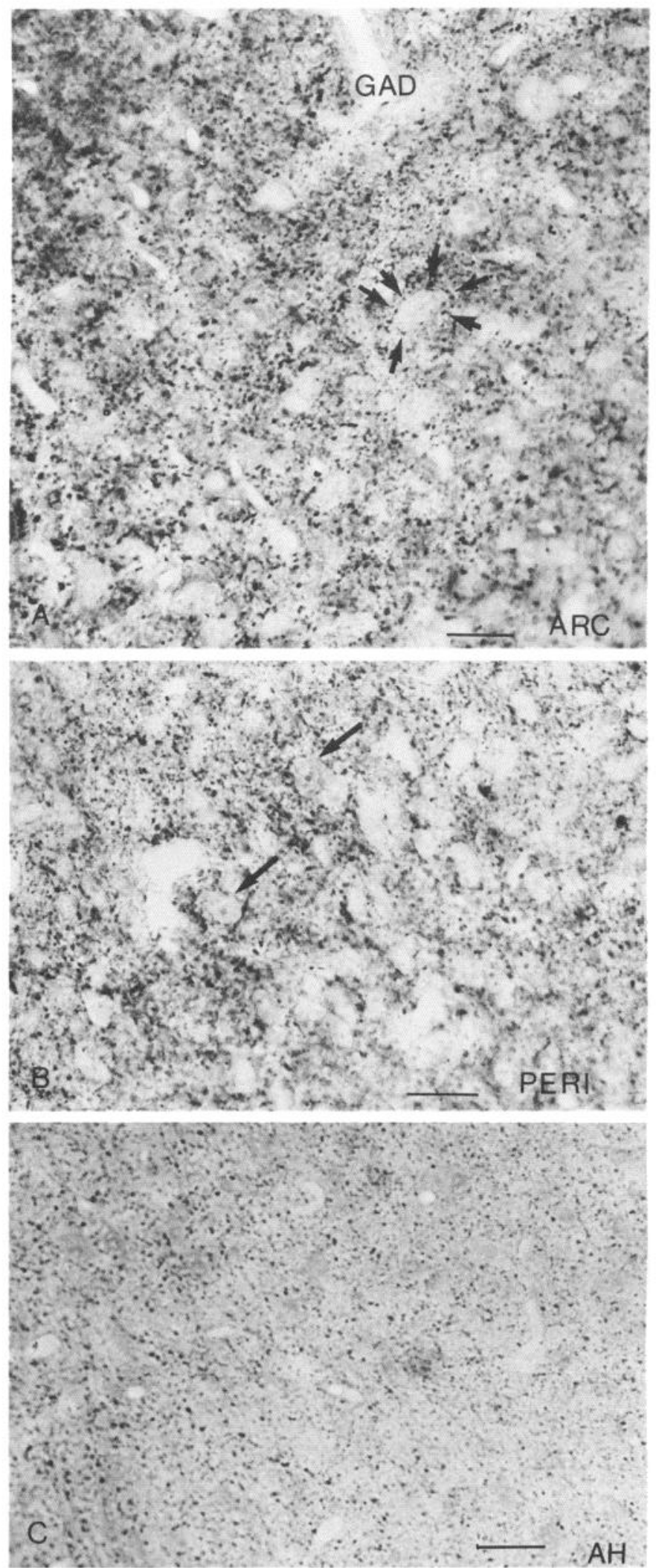

ventricular area $(P E R I)$, and $C$, the anterior hypothalamus $(A H)$. Arrows in $A$ indicate a number of GAD immunoreactive boutons surrounding an unlabeled cell body. Except where cells are clearly seen (arrows in $A$ and $B$ ), or in myelinated regions, immunoreactive axons are found in high density throughout the neuropil of the hypothalamus. Bars, $20 \mu \mathrm{m}$ $(A), 22 \mu \mathrm{m}(B), 30 \mu \mathrm{m}(C)$. 

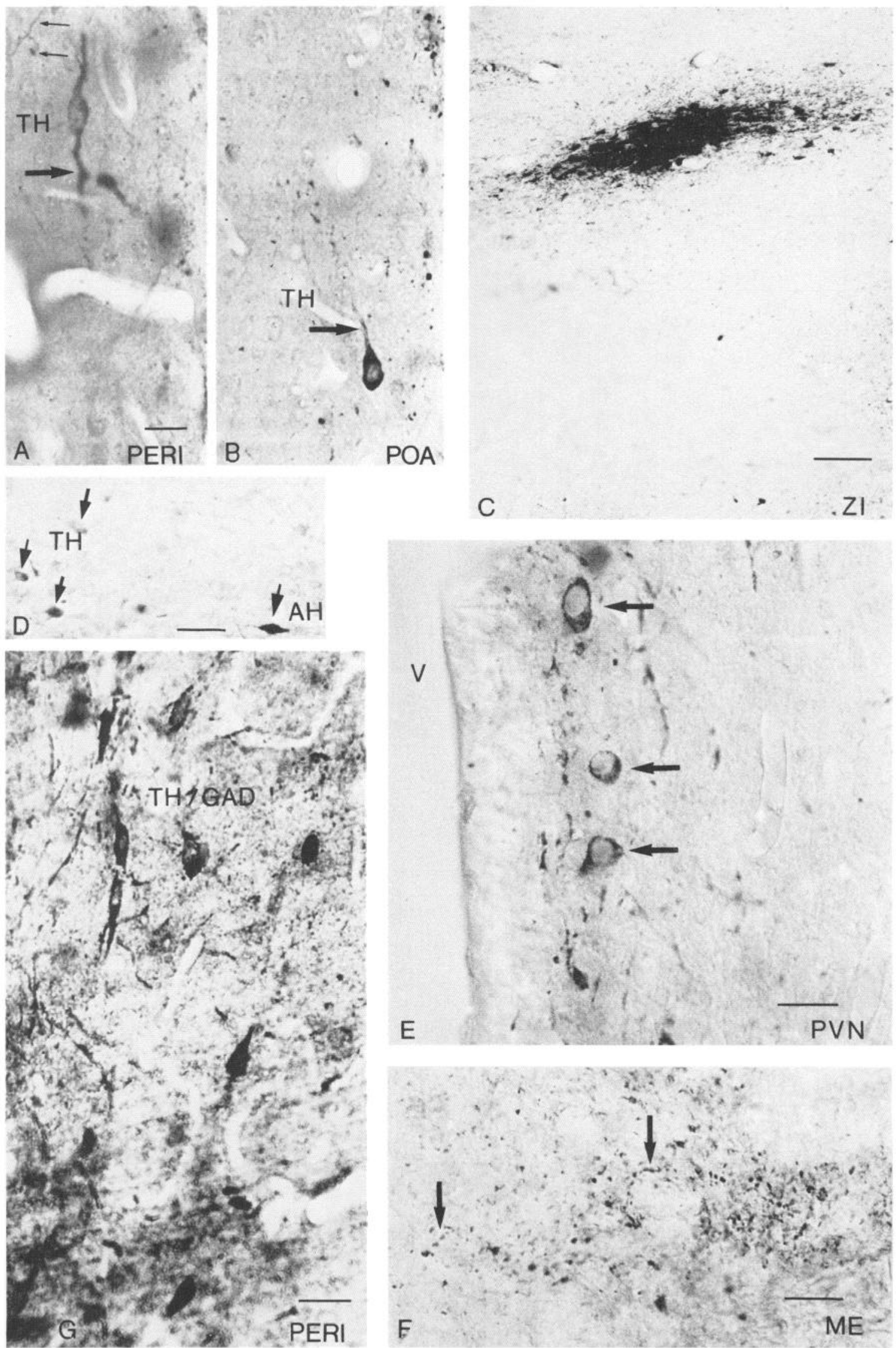

Figure 4. TH immunoreactivity: Silver-intensified gold. SIG-labeled TH immunoreactive neurons (arrows) are found in the following: $A$, Periventricular area $(P E R I)$, and $B$, preoptic area $(P O A)$. Bar for $A, B, 25 \mu \mathrm{m}$. $C$, Zona-incerta area $(Z I)$. Bar, $110 \mu \mathrm{m}$. $D$, Area dorsal to the supraoptic nucleus in the anterior hypothalamus $(A H)$. Bar, $40 \mu \mathrm{m}$. $E$, Medial paraventricular nucleus $(P V N)$ adjacent to the third ventricle $(V)$ : Silver reaction 


\section{Materials and Methods}

\section{Fixation}

Albino rats, generally males, were deeply anesthetized with pentobarbital (Nembutal) and perfused transcardially with $0.9 \%$ saline, followed by $4 \%$ paraformaldehyde and $0.1 \%$ glutaraldehyde in a $0.1 \mathrm{~m}$ phosphate buffer. The perfusion was terminated after $40 \mathrm{~min}$., the brain removed, and the hypothalamus dissected and immersed in fixative for $3 \mathrm{hr}$. To promote penetration of large immunoglobulin molecules into tissue, hypothalamic blocks were immersed first in $10 \%$ and then $20 \%$ sucrose, followed by freezing in liquid nitrogen. Coronal or horizontal sections, $30 \mu \mathrm{m}$, were cut on a Lancer Vibratome and subsequently washed in phosphate buffer.

\section{Peroxidase immunostaining}

To stain immunoreactive GAD axons, sections were first immersed in a blocking buffer containing $0.1 \%$ lysine, $0.1 \%$ glycine, $1 \%$ BSA, and $5 \%$ normal rabbit serum for $1 \mathrm{hr}$ in a TBS buffer $(20 \mathrm{~mm}$ Tris base, $0.9 \% \mathrm{NaCl}, 0.1 \% \mathrm{BSA}, \mathrm{pH} 8.2$ ). Sections were then incubated in sheep glutamate decarboxylase antiserum at a dilution of from $1: 1000$ to 1:3000 overnight. After subsequent washing in TBS, sections were incubated in a biotinylated rabbit anti-sheep immunoglobulin for $30 \mathrm{~min}$, washed, and then incubated in an avidin-biotin-HRP complex (ABC method of Hsu et al., 1981). After $30 \mathrm{~min}$ incubation in the $A B C$ complex, scetions were washed in TBS, and reacted with diaminobenzidine and hydrogen peroxide. Sections intended for single immunostaining were washed in phosphate buffer, osmicated, dehydrated in a graded series of ethanols and propylene oxide, and were then embedded in Epon.

\section{Colloidal gold immunostaining}

\section{Preparation of colloidal gold}

Colloidal gold particles of different sizes can be made by the reduction of gold chloride. Larger gold particles can be made by reduction with sodium citrate (Frens, 1973; Horisberger and Rosset, 1977), mediumsize particles with sodium ascorbate (Horisberger et al., 1978; Stathis and Fabrikanos, 1958), and the smallest particles (5 nm) with white phosphorus and ether (Faulk and Taylor, 1971; Horisberger and Rosset, 1977). A new procedure using allepo tannin has been recently described (Slot, 1984); in addition to being safer than the white phosphorus method, this approach allows gold particles from 3 to $17 \mathrm{~nm}$ diameter to be made by varying the amount of tannic acid used during reduction of the gold chloride.

\section{Protein adsorption to colloidal gold}

Colloidal gold has a negative charge and will adsorb quickly to many proteins (Horisberger, 1979; Romano et al., 1974; Roth, 1982; Roth et al., 1978). The $\mathrm{pH}$ of the solutions in which the adsorption takes place is important, and the proper $\mathrm{pH}$ is determined by the $\mathrm{pI}$ of the protein. For heterologous antisera, and for protein $\mathrm{A}$, we have used a $\mathrm{pH}$ of 9 in a $2 \mathrm{~mm}$ borax buffer adjusted to proper $\mathrm{pH}$ by $\mathrm{K}_{2} \mathrm{CO}_{3}$. Protein added to the gold will adsorb within a few seconds. Gold sols of different-sized gold particles have different colorations; the smaller gold particles have the general color of a dark red wine. Gold particles adsorbed to proteins are fairly stable, and the adsorbed protein prevents gold flocculation caused by addition of salt. Addition of $10 \%$ sodium chloride will cause unadsorbed particles to flocculate and precipitate; this can be determined by a color change from red to purple. A simple way to determine the correct amount of protein needed to stabilize gold particles is by addition of $100 \mu \mathrm{l}$ of $10 \%$ sodium chloride to $1 \mathrm{ml}$ of adsorbed gold. If any color change is seen, either more protein must be added to stabilize the gold, or the protein, for a variety of reasons, may not be suitable for adsorption to gold. Smaller gold particles require significantly more protein to stabilize than large particles. Furthermore, in our hands, some proteins seem to adsorb better to smaller gold particles than to larger gold particles; for instance, cholera toxin, used for tracing of neuronal pathways, adsorbed poorly to $40 \mathrm{~nm}$ gold, but stabilized $20 \mathrm{~nm}$ gold with little problem. After the correct amount of protein that will just stabilize the gold is determined, 10\% extra protein is added (DeMey et al., 1981). BSA (Sigma, fraction V) is added to a final concentration of $1 \%$. If the adsorption was successful, all the colloidal gold particles will be stabilized by protein, but excess protein (i.e., protein A or immunoglobulins) may not be adsorbed. To eliminate particle aggregates, a slow-speed centrifugation step is used; $5000 \times g$ is adequate for $5 \mathrm{~nm}$ gold, and slower speeds for larger gold. To eliminate unadsorbed protein A or IgGs, the solution is centrifuged at high speed. For $5 \mathrm{~nm}$ gold, DeMey (1983) recommends a $g_{\max }$ of 60,000 , while Slot and Geuze (1983) recommend a $g_{\text {av }}$ of 125,000 . The soft pellet is resuspended in $1 \%$ BSA in Tris-buffered saline, and the supernatant and any hard pellet are discarded. The speed of centrifugation is determined by the size of the gold particle. After two additional centrifugation steps, $0.1 \%$ sodium azide is added, and the solution is stored at $4^{\circ} \mathrm{C}$. Samples prepared in this way 3 years ago were found still viable for immunocytochemistry, although the signal-to-noise ratio was lower than for freshly prepared colloidal gold. The gold solutions can be stored in a lab freezer $\left(-20^{\circ} \mathrm{C}\right)$ if glycerol is added to prevent freezing.

One relatively simple way of standardizing colloidal gold solutions of monosize gold after adsorption to proteins is by measuring light absorption at $520 \mathrm{~nm}$ (Beckman model 24 spectrophotometer). Gold suspensions ( $1: 20$ of stock solution in 1\% BSA) are diluted to an O.D. of 0.25 for $5 \mathrm{~nm}$ gold, and 0.5 for $20 \mathrm{~nm}$ gold. Commercial colloidal gold adsorbed to proteins varies greatly in concentration, and appropriate working dilutions can be prepared after spectrophotometric analysis (DeMey, 1983).

Since colloidal gold adsorbed to primary antisera consistently gave poorer results than when adsorbed to a secondary immunoglobulin or protein A, a two-step immunostaining procedure was generally used for immunogold staining in the present scts of experiments.

\section{Immunostaining of tyrosine hydroxylase with gold particles}

Sections were incubated in one of two solutions intended to reduce nonspecific sticking of immunoglobulins to tissue sections. Both contained $0.1 \%$ glycine, $0.1 \%$ lysine, and BSA in a TBS buffer. Sections intended for use with a colloidal gold adsorbed to goat anti-rabbit immunoglobulin were immersed in blocking solutions also containing 5\% normal goat serum and $1 \% \mathrm{BSA}$, while sections intended for use with a protein A-adsorbed gold probe were put in blocking solutions containing 3\% BSA with no normal serum. After incubation in rabbit antityrosine hydroxylase antiserum overnight at a dilution of 1:1000, sections were washed thoroughly and put in gold adsorbed to protein A (Pharmacia) or to goat anti-rabbit IgG (Cappel). After $2 \mathrm{hr}$ agitation at room temperature, sections were washed in TBS repeatedly prior to silver intensification.

While gold of $20 \mathrm{~nm}$ and larger is visible as a faint pink coloration in the LM with immunostaining, this large-size gold particle penetrates tissue very poorly. Penetration is better with $10 \mathrm{~nm}$, and best with 3$5 \mathrm{~nm}$ gold. A simple way to assess the immunostaining with gold particles was to use a third immunoreagent conjugated to a fluorescent probe, either FITC or rhodamine (van den Pol, 1984a). If goat antirabbit IgG adsorbed to gold was used as a secondary reagent, FITCconjugated rabbit, or FITC rabbit anti-goat IgG was used to localize the gold probe (Fig. 2). Protein A adsorbed to gold, which can bind to two Fc sites on IgGs, could also be detected with rabbit IgG conjugated to FITC. Since free goat IgG or protein A was virtually eliminated through several centrifugation steps after adsorption to the colloidal gold, the FITC conjugate localized specifically the gold-adsorbed protein. If staining is not seen with the fluorescent marker, further ultrastructural study may not be useful.

\section{Silver intensification of metals}

Silver salts have been used for years to stain or intensify heavy metals in the nervous system (Brunk et al., 1966; Danscher, 1981; Haug, 1967, 1973; Pearson and O'Neill, 1958; Pihl, 1967; Tyrer and Bell, 1974). To

product is found throughout the cytoplasm of immunoreactive neurons (arrows) but not in cellular nuclei. Bar, $30 \mu \mathrm{m}$. $F$, SIG-labeled TH immunoreactive axons (arrows) in the median eminence $(M E)$. Bar, $8 \mu \mathrm{m}$. $G$. Periventricular hypothalamus $(P E R I)$; darkly stained $T H$ immunoreactive cells labeled with SIG are found in the same area as lightly stained $G A D$ immunoreactive axons stained with peroxidase. Bar, $15 \mu \mathrm{m}$. 
determine what parameters were optimal for intensification of colloidal gold particles, the use of nitrocellulose blots was invaluable. A routine test was devised where 2 or $5 \mu \mathrm{l}$ of different concentrations of different size colloidal gold particles were blotted on nitrocellulose (Schleicher and Schuell, PH 79, $0.1 \mu \mathrm{m}$ pore size). Samples were applied with a Ziptrol wire plunger in a $5 \mu \mathrm{l}$ micropipette (Drummond Scientific).

The labeled nitrocellulose was immersed in different silver intensification solutions, and the intensity of gold spots and the background staining on paper was compared.

The optimal intensification solution contained 1,4-benzenediol (hydroquinone, Sigma H-9003), a colloidal suspension of gum acacia (gum arabic, Sigma G-9752) in a citrate buffer containing silver nitrate (Fisher S-181). This solution was similar to that used by Haug (1973) for routine (nonimmuno) silver staining of the brain. The $\mathrm{pH}$ of the buffer was about 4 . The intensification solution should be made immediately before use, with the silver nitrate added as the final step just before immersion of the sections in it. As gum arabic may take several hours to dissolve, we generally made large batches ( 1 part gum acacia, 2 parts $\mathrm{H}_{2} \mathrm{O}$ ) and kept them frozen in $20 \mathrm{ml}$ vials until needed. Some of the older papers (Haug, 1973) have indicated an advantage of using large pieces of acacia and mixing it for several days before use. We found that the commercially prepared powder (Sigma) worked sufficiently well and dissolved in a few hours. Silver lactate (Danscher, 1981; Holgate et al., 1983) can be substituted for silver nitrate, but as it was more expensive and dissolved more slowly, silver nitrate was generally used.

The final silver-intensifying solution was similar to those described previously (Danscher, 1981; Haug, 1973). Four stock solutions were made the day of the experiment: (1) $2 \mathrm{M}$ citrate buffer (25.5 gm citric acid, $23.5 \mathrm{gm}$ trisodium citrate, in $100 \mathrm{ml} \mathrm{H}_{2} \mathrm{O}$ ). (2) $5.6 \%$ hydroquinone in $\mathrm{H}_{2} \mathrm{O} ; 0.75 \%$ silver nitrate in $\mathrm{H}_{2} \mathrm{O}$; and gum arabic (described above).

To $12 \mathrm{ml}$ gum acacia solution, $2 \mathrm{ml}$ of citrate buffer was added and mixed. Then $3 \mathrm{ml}$ of the hydroquinone solution was mixed in. As a final step, $3 \mathrm{ml}$ of silver nitrate was added. As with all silver solutions, care should be taken to use clean glass or plastic dishes and clean tools; in general, metallic tools should be avoided.

In place of a colloidal suspension of gum arabic, other substances were substituted, including gum tragacanth (Sigma G-85), 5\% BSA, and Lipshaw embedding matrix. Alternatively, $4 \%$ gelatin in a $40 \%$ ammonium nitrate buffer (Gorcs et al., 1979) was also effective in gold intensification; to $10 \mathrm{ml}$ of this buffer was added $1 \mathrm{ml}$ of $0.75 \%$ silver nitrate and $0.5 \mathrm{ml}$ of $5.6 \%$ hydroquinone.

In addition to colloidal gold, other heavy metals bound to immunoglobulins were compared in attempts to find an effective marker that could be used for double immunostaining. A number of metallic compounds were used, bound either to BSA or immunoglobulins. Additionally, homocysteine thiolactone was used to increase the binding of gold, silver, palladium, or platinum metal salts to proteins (Shall and Barnard, 1969). Methyl picolinimidate (Benisek and Richards, 1968) and osmium and thiocarbohydrazide (Sternberger, 1979; Sternberger et al., 1966) were also used to increase metal deposition on proteins.

\section{Silver-intensified gold procedure for immunostaining of a single antigen}

Sections intended for the SIG reaction were stained with primary antiserum against tyrosine hydroxylase (1:1000) followed by a secondary ligand adsorbed to colloidal gold, as described above. Gold probes of $5,10,15,20$, and $40 \mathrm{~nm}$ diameter were used. After $2 \mathrm{hr}$ in the goldadsorbed protein solution, sections were thoroughly rinsed in TBS and were then ready for silver intensification. After washing in $0.2 \mathrm{M}$ citrate buffer to remove the TBS buffer, sections were placed in the silverintensification solution in a $20 \mathrm{ml}$ scintillation vial. Vials were wrapped in aluminum foil and placed on a small platform standing on springs, which was agitated automatically every $10 \mathrm{sec}$. Incubation times in the silver nitrate intensification solution were critical, varying from $3 \mathrm{~min}$ to 2 hr. Doing several repetitions of the silver-staining procedure with different tissue sections was helpful. Alternatively, sections could be inspected under dim light during the staining procedure, after which they could either be rinsed in buffer or incubated longer. If the brown intensification solution began to turn gray or black, sections were either removed and washed in buffer or were placed in freshly made silverintensifying solution. The intensification step was usually run at room temperature; if the solution was cooled down prior to tissue incubation, sections could be left in the solution at $4^{\circ} \mathrm{C}$ for several hours or, with a reduction in silver nitrate or hydroquinone concentrations, overnight.

The optimal procedure for intensifying immunogold-labeled sections consisted of several preliminary intensifications with test sections from the same area of the brain; the optimal time could be determined before all the remaining sections were stained. Optimally immunolabeled sections with a minimum background were obtained with intensifications stopped before the intensifying solution turned dark. Overintensification occurred on the sections when they had been left in an intensifying solution that had darkened. Overintensified sections contained nonspecific cellular and background darkening at the LM level and an increased density of nonspecific particles randomly distributed throughout thin sections at the ultrastructural level. After the silver-intensification step, sections were rinsed in either the citrate buffer or water. Sections could then be put back in a phosphate buffer prior to osmication, dehydration through an ascending series of ethanols and propylene oxide, and embedding in Epon.

\section{Double pre-embedding immunostaining: SIG and peroxidase}

Since our primary question in these studies focused on GABA innervation of dopamine neurons, the order of staining was compared. In one series, TH was stained with the SIG procedure above, and then GAD was stained with peroxidase. In another series, GAD was stained with peroxidase, and then TH was subsequently stained with the SIG procedure. The steps for double-labeling are outlined in Table 1.

\section{Antisera}

Previous work describing the specificity of sheep anti-GAD (Oertel et al., 1981 a, b; Tappaz et al., 1983) and rabbit anti-GABA antisera (Hodgson et al., 1985; Somogyi et al., 1985) have been published.

As we previously reported, the $\mathrm{TH}$ antiserum recognized a single molecular weight band; specificity was further tested by precipitation of enzyme activity, immunoblotting, and precipitation of cell-free translation products. Absorption of antiserum with purified TH abolished staining, while addition of bovine dopamine beta-hydroxylase had no apparent effect on immunostaining (van den Pol et al., 1984). Deletion of either primary antisera resulted in no staining for the homologous antigen. Substitution of other antisera stained neurons and axons in different hypothalamic regions.

Additional controls are described in Results.

\section{Results}

\section{Silver intensification of "metallic" proteins}

Dot blots of different-sized colloidal gold particles all turned black or gray after intensification with silver nitrate (Fig. 1, $A$ $C$ ). Attempts to obtain suitable intensification of proteins treated with a variety of metallic salts including palladium, platinum, cobalt, and gold chloride did not work well compared with colloidal gold. The most promising of these compounds was protein treated with homocysteine thiolactone and silver nitrate. However, even this could not be intensified to the same level as a dilute solution of colloidal gold (Fig. $1 C$ ), and the potential advantage of having a smaller-sized reagent (silver/IgG) was offset by its less promising intensification. A number of variants of silver deposition procedures have been used to localize naturally occurring or experimentally administered heavy metals. When gum arabic was compared with other protective colloids, a colloidal suspension of gum arabic gave a higher signal-tonoise ratio on dot blots than BSA, gum tragacanth, Lipshaw embedding matrix, or macrocolloidal suspension; while silver intensification could be achieved in the presence of any of these substances, the sensitivity was greatly reduced and background staining increased rapidly. Other colloids that might be substituted for gum arabic are Dextran 80 (Pihl, 1967) or gelatin (Pearson and O'Neill, 1946). Gum arabic was found superior to Dextran, polyethelene glycol, and polyvinylpyrolidone by Brunk et al. (1968) for silver intensification.

The ability of different-sized colloidal gold particles to penetrate and stain tyrosine hydroxylase immunoreactive neurons in the hypothalamus and ventral tegmental/substantia nigra area was compared. In $30 \mu \mathrm{m}$ Vibratome sections frozen previously in liquid nitrogen and intensified after immunostaining with the 

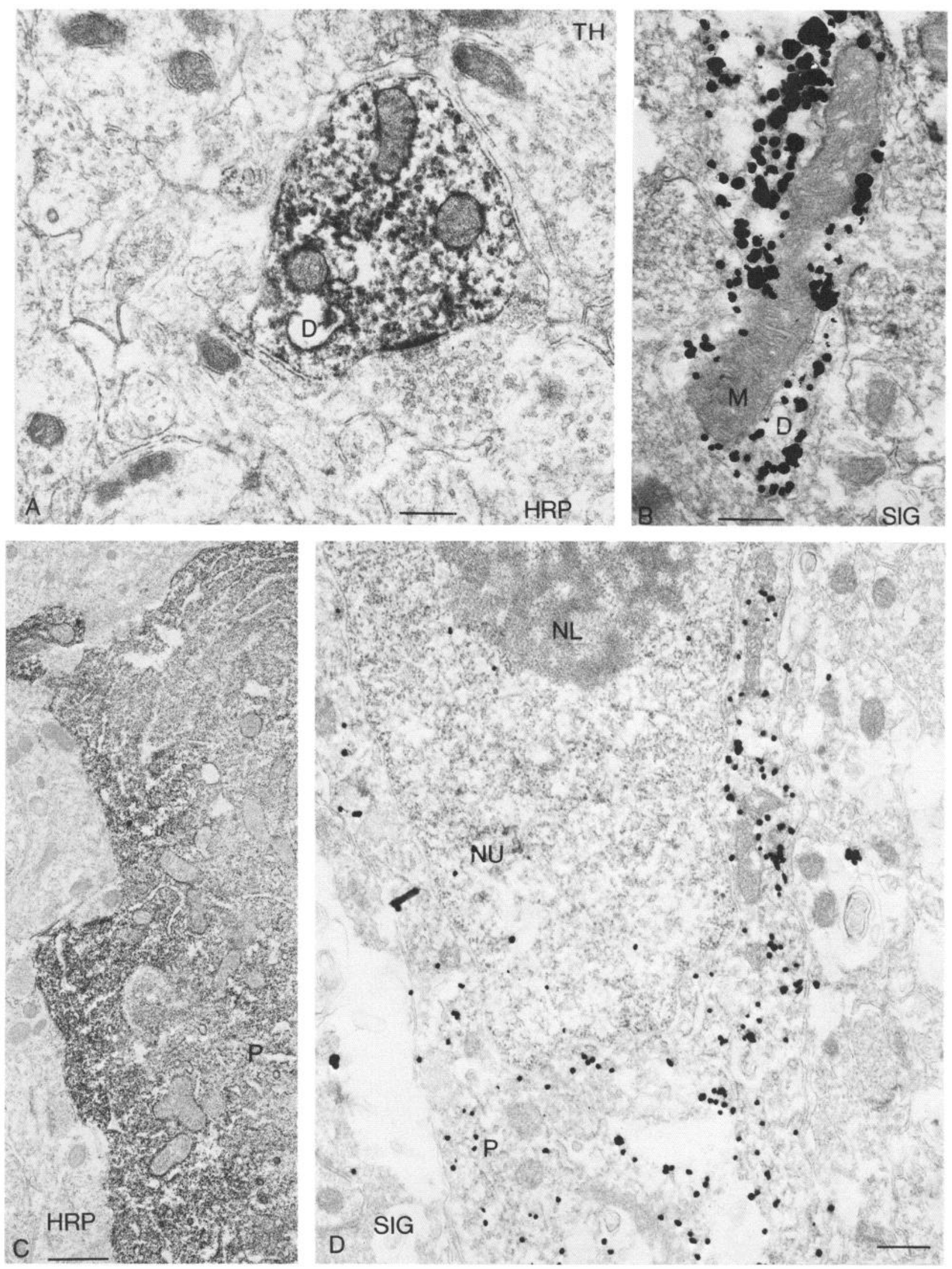

Figure 5. TH immunoreactivity: HRP or SIG. TH immunoreactive dendrites $(D)$ and perikarya $(P)$ stained with peroxidase $(H R P ; A, C)$ or silver $(S I G ; B, D)$ can be compared. In neither procedure does the reaction product stain inside mitochondria $(M)$. The apparent staining of the outer membrane of the mitochondria with HRP may be an artifact of the procedure, rather than a bona fide localization of the TH antigen. $N U$, Nucleus; $N L$, nucleolus. Bars, $250 \mathrm{~nm}(A), 200 \mathrm{~nm}(B), 300 \mathrm{~nm}(C)$, and $225 \mathrm{~nm}(D)$. 


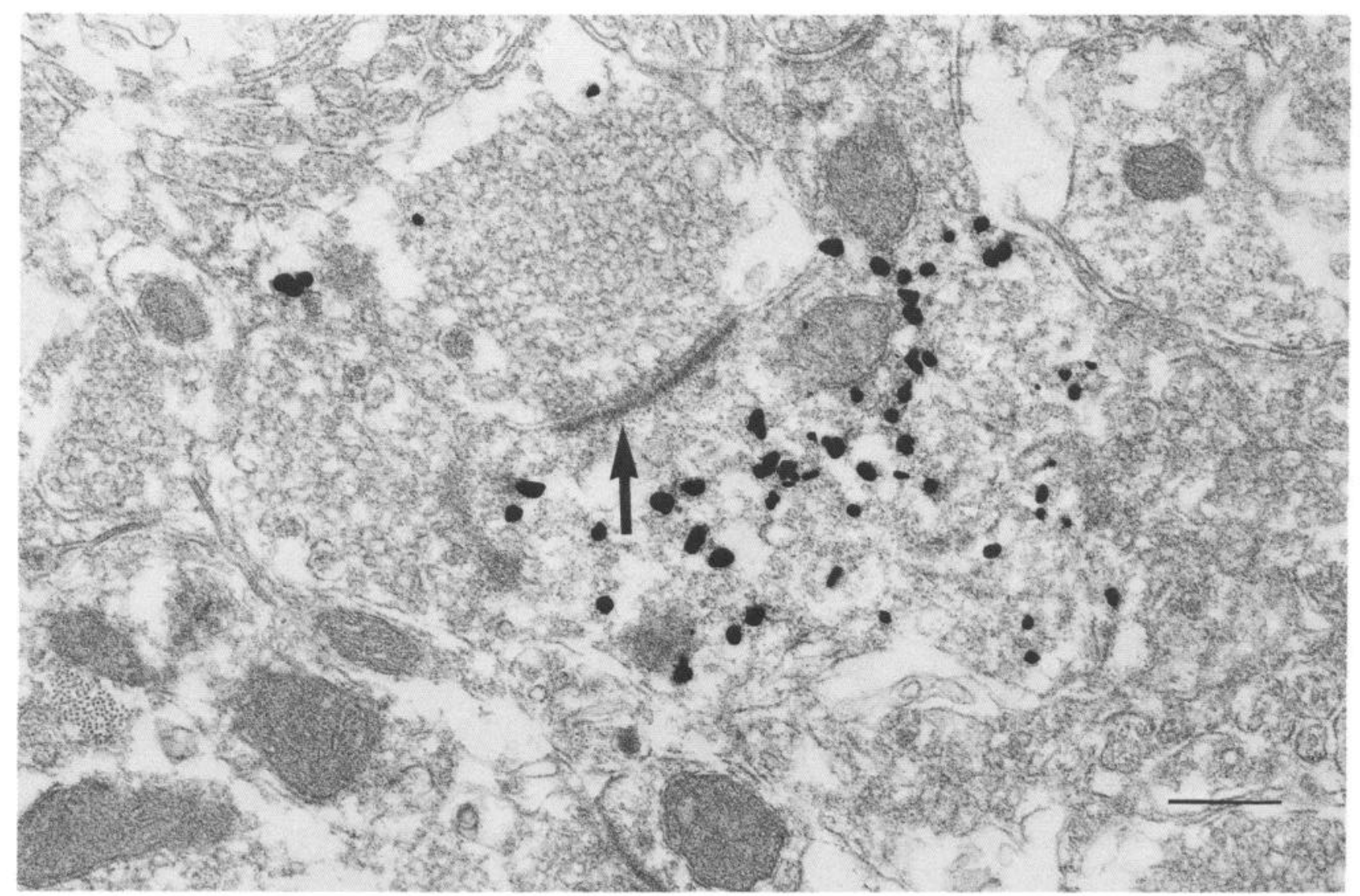

Figure 6. TH immunoreactive dendrite: SIG. The labeled dendrite receives an unlabeled asymmetrical synapse (arrow) from an axon filled with clear vesicles and with an occasional larger, dense-core vesicle. No GAD peroxidase staining is seen in this photomicrograph. While the fixation is not optimal, organelles can be identified, particularly synaptic vesicles. Membrane damage, necessary for penetration of the colloidal gold and other immunoreagents, is evident. In serial sections, silver particles consistently labeled this single post-synaptic dendrite. Bar, $250 \mathrm{~nm}$.

silver nitrate procedure, neuronal staining was not found with 40 or $20 \mathrm{~nm}$ gold particles. Positive immunostaining was found with 10 and $5 \mathrm{~nm}$ particles, and was best with the $5 \mathrm{~nm}$ colloidal gold. The $20 \mathrm{~nm}$ gold particles stained $\mathrm{TH}$ immunoreactive neurons in 5 or $10 \mu \mathrm{m}$ sections embedded in paraffin or polyethylene glycol, which was seen as a faint pink coloration. The 5 and $10 \mathrm{~nm}$ particles were not visible with light microscopy, but their presence could be detected with the use of a tertiary fluorescent probe (Fig. 2) prior to silver intensification.

\section{TH and GAD immunoreactivity in the medial hypothalamus}

\section{GAD immunoreactivity (peroxidase)}

With single-labeling experiments, axons immunoreactive with sheep antiserum against rat GAD were found with terminal boutons in all hypothalamic regions in which TH immunoreactive perikarya and dendrites were found. Axons were also located in the same areas with rabbit antisera against GABA. Examples of dense GAD immunoreactivity in axons and boutons from the periventricular area, arcuate nucleus, and anterior hypothalamus are shown in Figure 3. Labeling with GAD or GABA was much greater throughout the hypothalamus than in thalamic areas. Without administration of axonal transport blocking agents like colchicine, and with the antisera dilutions used for double staining, GAD immunoreactive somata and dendrites were not generally found in the medial hypothalamus.

\section{TH immunoreactivity (SIG)}

TH immunoreactive neurons stained with the SIG procedure were found in all the same regions as TH immunoreactive cells stained with peroxidase (van den Pol et al., 1984) with the ABC method (Hsu et al., 1981) or PAP method (Sternberger, 1979), including the periventricular, preoptic, zona incerta-dorsal hypothalamic regions, and the arcuate and medial paraventricular nuclei (Fig. 4). Without silver intensification, TH immunoreactive cells immunostained with $5 \mathrm{~nm}$ colloidal gold were not visible with light microscopy. During silver intensification, cells turned first yellow, then brown, and finally black, corresponding to an increased deposition of silver on colloidal gold particles.

Ultrastructurally, the silver-intensified gold particles were found throughout the cytoplasm of perikarya, dendrites, and axons in the medial hypothalamus (Fig. 5, $B$ and $D$ ) and in the median eminence. Unlike peroxidase reaction product, which sometimes seemed to stick to outer membranes of cytoplasmic organelles (Fig. 5, $A$ and $C$ ), the silver particles did not seem to show such a localization. Silver particles were generally not found in large organelles such as mitochondria, nuclei, and nucleoli. Serial sections through $\mathrm{TH}$ immunoreactive processes showed SIG deposits in the same structures in all sections (Fig. $6)$. The size of the silver-intensified gold particles varied, depending on the degree of silver intensification. Intensified particles were generally too large to allow any resolution over smaller organelles such as endoplasmic reticulum or vesicles.

\section{Double labeling for GAD and TH}

Ultrastructurally, in the same thin section TH immunoreactive dendrites and somata were found stained with large particles of silver-intensified gold and GAD immunoreactive axonal boutons were revealed with electron-dense matrix typical of osmicated HRP reaction product (Fig. 7). GAD immunoreactive axons made synaptic contact with dendrites and somata with a 
typical symmetrical-type synaptic specialization; the presynaptic GAD immunoreactive axon contained small clear vesicles and an occasional dense core vesicle. GAD immunoreactive axons, stained with peroxidase, were also found in synaptic contact with $\mathrm{TH}$ immunoreactive perikarya and dendrites stained with the SIG method. Since all regions of the hypothalamus that contained TH immunoreactive cells also had large numbers of GAD immunoreactive boutons, several of these areas were studied ultrastructurally. Somata and proximal dendrites of neurons in the arcuate nucleus were labeled with the SIG procedure; immunoreactive dendrites labeled with silver had the same general characteristics of previously described dendrites stained with peroxidase (van den Pol et al., 1984), and the SIG immunoreactive dendritic arbor was oriented in the same general direction as Golgi-impregnated cells in the same part of the nucleus (van den Pol and Cassidy, 1982). In the periventricular region of the hypothalamus (A14), and reaching into the medial paraventricular nucleus, TH immunoreactive cells were stained, and proximal dendrites in coronal sections were often situated parallel to the wall of the third ventricle. The TH immunoreactive cells with the largest dendrites were found in the caudal part of the dorsomedial hypothalamus/zona incerta group, and immunoreactivity could be found in dendrites at considerable distances $(>100 \mu \mathrm{m})$ from the perikaryon. In all regions examined ultrastructurally, including the arcuate, medial paraventricular, and dorsomedial nuclei and the periventricular area in the anterior hypothalamus, GAD immunoreactive boutons consistently made contact with $\mathrm{TH}$ immunoreactive dendrites and perikarya (Figs. 8 and 9). When serial section reconstruction was attempted on several SIG immunoreactive dendrites, peroxidase immunoreactive boutons were consistently found contacting the dendrites. To increase the percentage of neural structures labeled with GAD or TH, tissue blocks were frozen and thawed prior to immunocytochemical reaction. This, in addition to the hydrogen peroxide used in the DAB reaction and the low $\mathrm{pH}$ of the silver-intensification solution, resulted in some deterioration of ultrastructure preservation; nonetheless, in all regions studied, synaptic membrane specializations could be found (e.g., Figs. 8, $A, B$, and $E$ and $9 A$ ). Tissue that was intensified for only a short period generally showed better preservation than tissue intensified for a longer time.

Sections treated only with peroxidase and DAB showed no particulate staining indicative of the SIG reaction product. Conversely, sections stained only with the SIG procedure showed no diffuse reaction product indicative of peroxidase labeling. Sections immunostained with silver-intensified colloidal gold and then treated with diaminobenzidine showed no diffuse DAB reaction product. Deletion of the primary antisera eliminated staining of the homologous antigen. As with fluorescent and peroxidase immunocytochemistry, overstaining with the SIG procedure resulted in a nonspecific deposition of silver particles throughout the tissue.

In addition to $\mathrm{TH}$, other neurotransmitter-related antigens in the hypothalamus have been stained with the SIG procedure; these include neurophysin, GAD, LHRH, somatostatin, and prolactin.

\section{Silver intensification of peroxidase reaction product}

As indicated in Table 1, when double staining with the SIG and $\mathrm{ABC}$ method, the SIG procedure should be used with the first primary antibody and peroxidase with the second. This order was necessary because the same silver nitrate intensification solution worked not only to increase the size of gold particles, but also to intensify the diaminobenzidine HRP reaction product. Not only did the intensifying solution cause the HRP reaction product to turn from a light brown to black, but on close inspection of TH immunoreactive neurons stained with the $\mathrm{ABC}$ method followed by silver intensification, the increased opacity of the reaction product was found to be caused by a granular

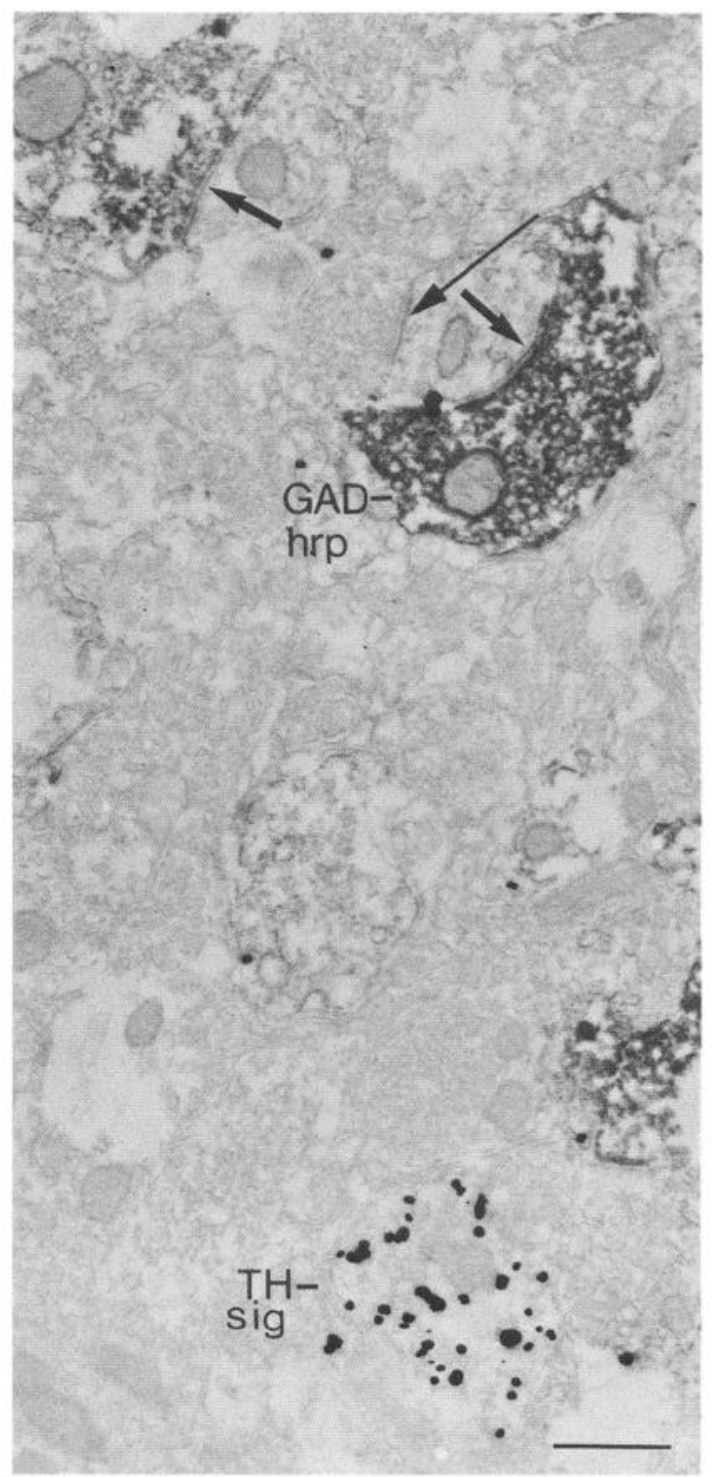

Figure 7. In a section from the arcuate nucleus, a single $T H$ immunoreactive dendrite labeled with the $S I G$ procedure is seen in the same thin section as two $G A D$ immunoreactive boutons making synaptic contact (short arrows) with unlabeled dendrites. One unlabeled dendrite is also in synaptic contact with a second unlabeled axon (long arrow). No peroxidase label is seen in the SIG-stained dendrite, and, vice versa, peroxidase-stained axons are free of silver particles. Photomicrograph is printed light to facilitate identification of HRP and silver particles. Bar, $450 \mathrm{~nm}$.

precipitate, as seen both with high-magnification light microscopy (Fig. 10) and electron microscopy (Fig. 10). The size and number of particles distributed within the HRP reaction product was influenced by the duration of silver intensification. In thin sections of the arcuate nucleus that had been stained with the silver-intensified HRP method, all TH immunoreactive boutons, dendrites, and somata had a granular-particulate peroxidase reaction product that could be distinguished from nonintensified HRP with both light and electron microscopy, but was best differentiated with ultrastructural examination. Reversal of the staining sequence in Table 1 may result in a consistent double-labeling (HRP reaction product and silver particles) in the immunoreactive profiles stained first; only the second antigen would have a singel label-in this case, the silver-intensified gold. 

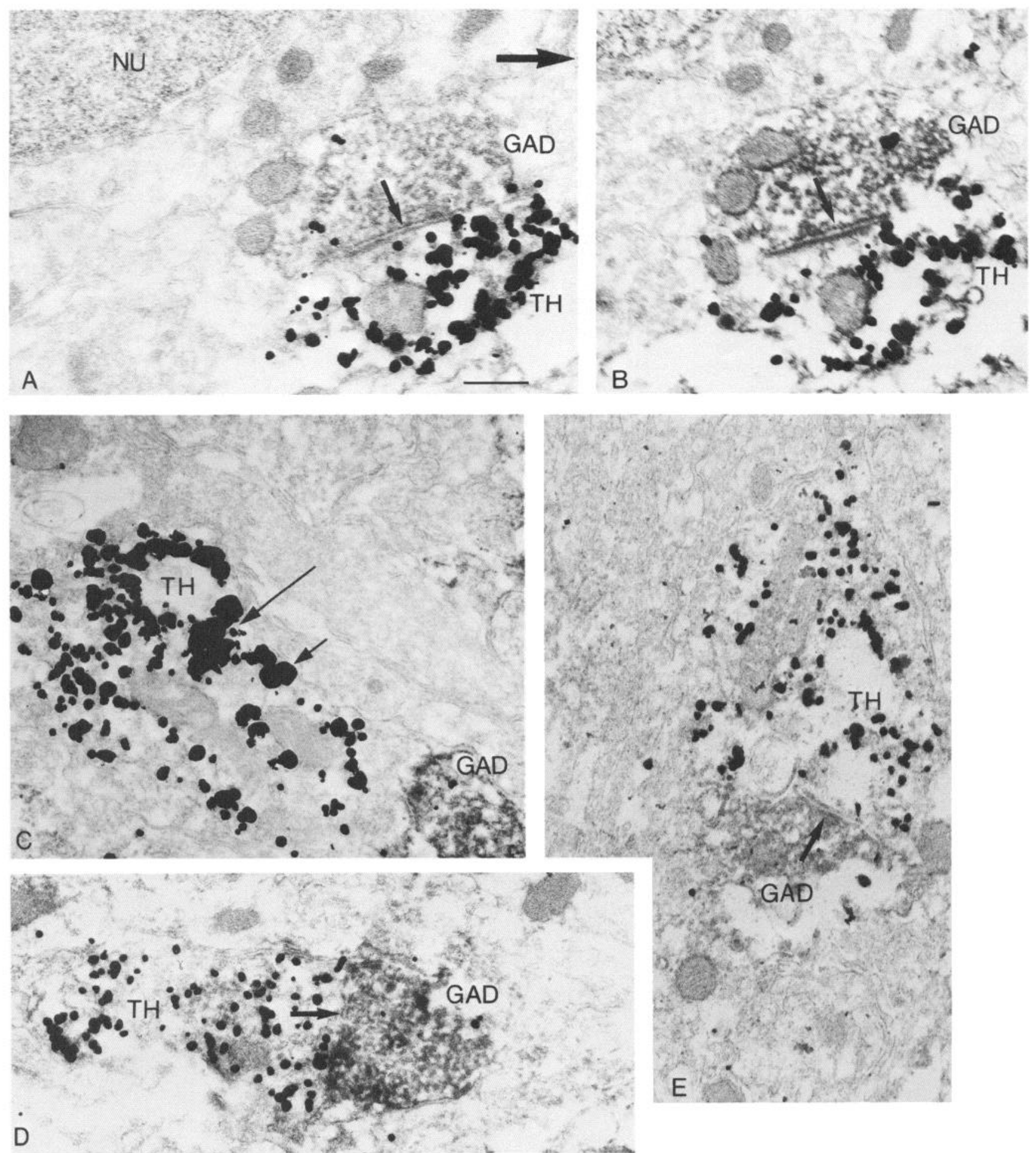

Figure 8. GAD boutons terminate on TH dendrites. $A$ and $B$, Serial sections of a SIG-labeled $T H$ immunoreactive dendrite in synaptic contact with a peroxidase-labeled $G A D$ immunoreactive axon in the hypothalamic arcuate nucleus $(N U)$. $C$, Close apposition of SIG-stained dendrite with HRP-stained axon. Note the large size of some of the silver-intensified gold particles (arrows). $D$ and $E$, In the periventricular area of the hypothalamus, SIG-labeled TH immunoreactive dendrites are frequently found in possible synaptic contact (arrows) with HRP-labeled GAD immunoreactive axons. Bar, $300 \mathrm{~nm}$.

\section{Discussion}

Immunocytochemistry

Silver intensification of colloidal gold adsorbed either to the bacterial Fc receptor protein $\mathrm{A}$ or to immunoglobulins was used in combination with peroxidase for double ultrastructural immunocytochemistry. Critical for double immunostaining is the reduction or elimination of cross-reactivity between the first and second series of reagents, used respectively to stain the first and second antigen of interest. With the silver intensification of the gold, as described here, the silver precipitated around the 
colloidal gold particle would cover not only the gold, but also the adsorbed protein $\mathrm{A}$ or immunoglobulin, thereby reducing or eliminating further cross-reaction when the SIG procedure is used for the first antigen. Another critical factor in using the SIG procedure prior to HRP is that the silver intensification described here also intensifies the HRP reaction product, and gives it a particulate quality, recognizable both with light and electron microscopy. The particulate nature of the HRP after silver intensification cannot easily be discriminated from an immunoreactive profile containing both silver-intensified gold and peroxidase. The particulate nature of the silver-intensified peroxidase reaction product can itself be used as a second immunolabel in conjunction with nonintensified HRP. If one is fortunate enough to have two primary antisera raised in different species, through using appropriate secondary antisera cross-adsorbed to solid-phase IgG, cross-reaction of two series of immunoreagents can be eliminated. On the other hand, the procedure used here will work with two primary antisera raised in the same animal, owing in part to the masking of immunoreagents by a silver shell around the gold, and in part to protein A specificity. The use of protein A adsorbed to colloidal gold as a secondary reagent to stain the first antigen in double immunostaining can also serve to reduce cross-reactivity between two series of immunoreagents, because its second available Fc binding site can be blocked with the addition of immunoglobulins. Protein A has been used outside the nervous system for double labeling of two antigens with two different-size gold probes on thin sections (Geuze et al., 1981; Roth, 1982).

The SIG procedure was used in the present sets of experiments primarily to stain $\mathrm{TH}$ immunoreactive neurons; the same protocol has also been used to stain a number of other hypothalamic neurotransmitter-related antigens, including somatostatin, prolactin, neurophysin, GAD, and LHRH. The silver-intensified gold procedure stained TH neurons in all regions of the hypothalamus in which previous studies had reported immunoreactive neurons, confirming previous reports based on fluorescence or peroxidase immunochemistry (Bjorklund et al., 1975; ChanPalay et al., 1984; Fuxe, 1964; Swanson et al., 1981; van den Pol et al., 1984) and attesting to the sensitivity of the SIG method for light microscopy.

The SIG/HRP double-labeling method described here is suitable for the examination of two antigens located in different cells; the procedure may not be suitable for studies of colocalization of two antigens in the same cell. In the present study, in some cases, but not all, TH immunoreactive dendrites labeled with SIG also had a slight increase in general cytoplasmic density, similar to that seen after the DAB reaction. This may represent colocalization of dopamine and GABA in the same neurons in the arcuate nucleus, as recently described (Everitt et al., 1984), or may result from some minor cross-reaction between DAB and silver reagents. A different double-labeling procedure combining pre-embedding peroxidase with post-embedding colloidal gold staining also can be used with primary antisera from the same species (van den Pol, 1983, 1984a, 1985); crossreactivity is eliminated by the plastic used between the two series of immunostaining steps.

\section{GABA innervation of hypothalamic dopaminergic neurons}

GAD and GABA immunoreactive fibers are found throughout the hypothalamus, as suggested in the present study using antiserum against GAD and GABA, and in previous biochemical or immunocytochemical studies with GAD antiserum (Tappaz et al., 1982, 1983; Vincent et al., 1982). In all areas containing TH immunoreactive neurons, GAD and GABA immunoreactive axons are numerous. $\mathrm{TH}$ immunoreactive neurons in the periventricular area (A14), arcuate nucleus (A12), and caudal hypothalamus (A11) were surrounded by axons containing GAD
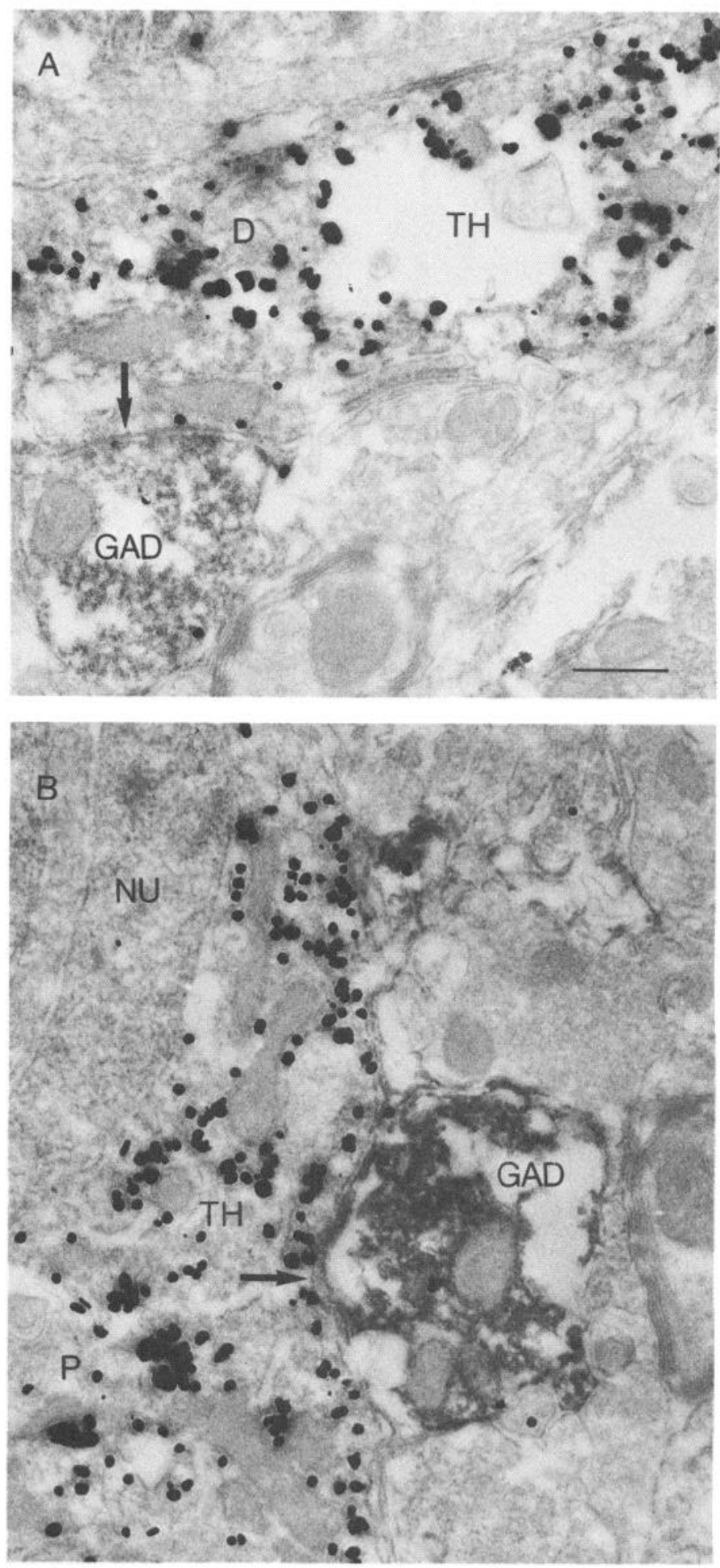

Figure 9. GAD immunoreactive axon in contact (arrows) with $T H$ immunoreactive dendrite, $D$, in the paraventricular nucleus $(A)$ and perikaryon, $P(B)$. Symmetrical synaptic specialization in $A$ is indicated at arrow. SIG reaction product seems restricted to cytoplasm and is not found in the nucleus $(N U)$. Bar, $275 \mathrm{~nm}$.

immunoreactivity. In a previous report (van den Pol, 1984b, 1985a), GAD immunoreactive boutons were also found in synaptic contact with TH immunoreactive neurons in the dorsomedial hypothalamus (A13). Similarly, in the substantia nigra, the origin of a massive dopamine projection to the striatum, dopaminergic neurons are contacted by GAD immunoreactive 


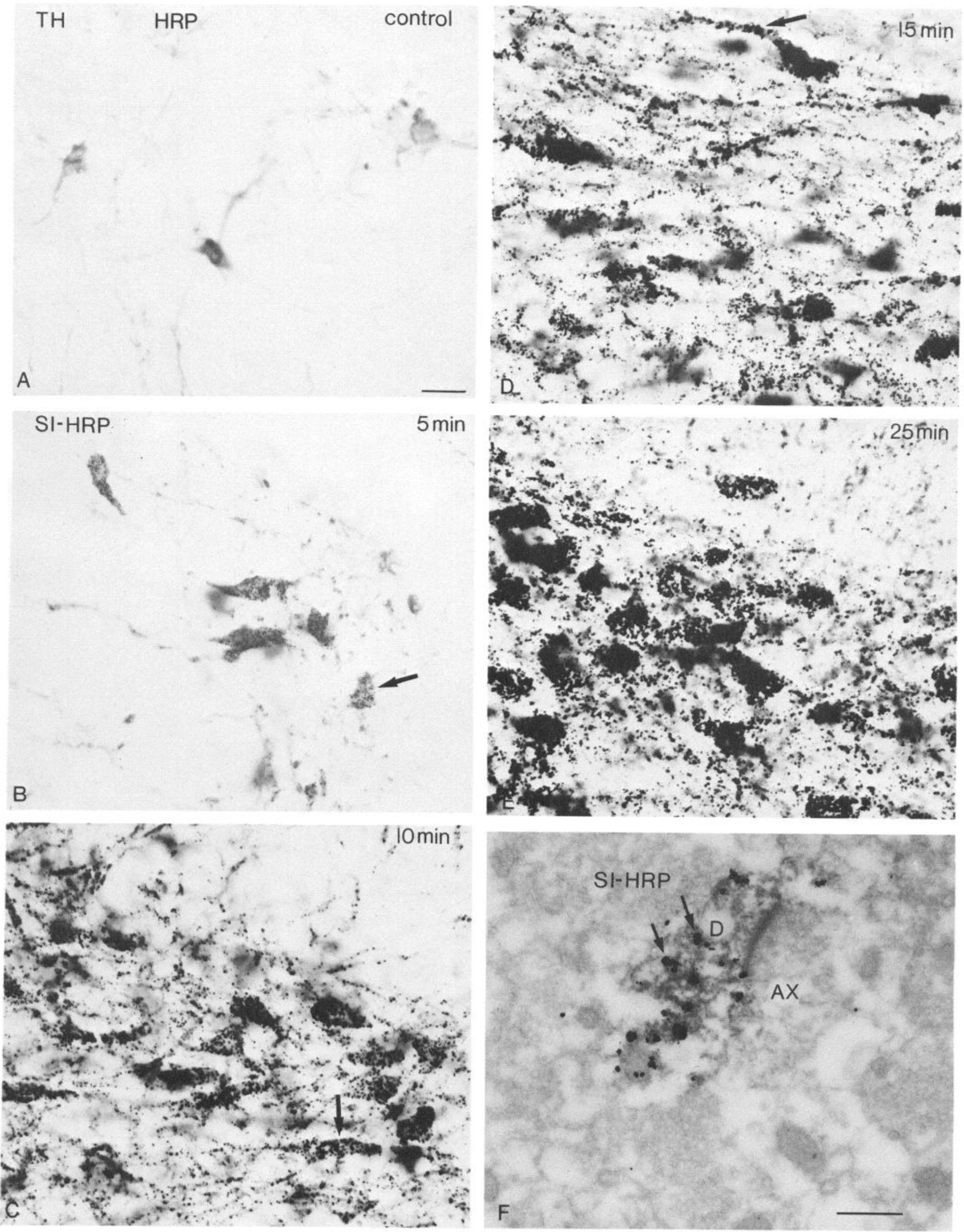


boutons forming symmetrical synapses (van den Pol et al., 1985). All regions containing dopamine neurons that we have examined also contain frequent GAD immunoreactive boutons, suggesting that direct GABA modulation may be a common feature of dopaminergic neurons in many areas of the brain. An additional question that arises is whether axons containing GAD immunoreactivity are selectively terminating on dopamine neurons or whether GAD immunoreactive neurons are simply so frequent that they terminate on many or all neurons in the areas where large numbers of boutons are located. In a study with antisera against 25 different putative neurotransmitters in the hypothalamus, boutons stained with GAD or GABA were the most widespread and appeared at the greatest density both with light (van den Pol and Tsujimoto, 1985) and electron (A. N. van den Pol, unpublished observations) microscopy. With a different double immunolabeling protocol of pre-embedding peroxidase followed by post-embedding colloidal gold, GAD and GABA immunoreactive boutons were found to synapse on neurophysin immunoreactive neurosecretory neurons of the supraoptic nucleus (van den Pol, 1985b).

The role of GABA in neuroendocrine events can be divided into three categories: direct modulation at the level of the pituitary, modulation of dopamine neurons of the hypothalamus, and modulation of other nondopaminergic neurons that participate in endocrine regulation. The possibility that GABA is itself a pituitary tropin has been the subject of a wide range of in vivo and in vitro physiological studies, and is reviewed elsewhere (Elias et al., 1982; Ondo and Pass, 1976; Racagni et al., 1982; Tappaz, 1984). In brief, GABA may function as a prolactininhibiting factor via GABA receptors in the pituitary, although with less potency than dopamine (Enjalbert et al., 1979; Schally et al., 1977). Injections of GABA, GABA agonists, and GABA antagonists into the cerebrospinal fluid have been reported to influence not only prolactin, but also gonadotropins, adrenocorticotropin, growth hormone, and thyrotropin (Elias et al., 1982; Racagni et al., 1982; Tappaz, 1984). The literature is too voluminous to review here, but it does underscore the importance of GABA in the brain as a modulator of hormones of the anterior pituitary and, through the pituitary, of endocrine organs including the gonads, thyroid, and adrenal.

In the present study we found a direct synaptic innervation of dopamine neurons in the medial hypothalamus by GABAergic axons, indicating an inhibitory role of GABA in the modulation of median eminence dopamine. The function of the GABAergic innervation of presumed dopamine neurons in the hypothalamus can be viewed in light of the known functions of the dopamine neurons themselves in this region. Some of the axons from neurons in the region of the arcuate nucleus terminate in median eminence (Bjorklund et al., 1975; Fuxe, 1964; Szentagothai et al., 1972; van den Pol and Cassidy, 1982), where released dopamine is carried by the portal vascular system to the anterior pituitary; there, dopamine serves as a neurohormone, causing inhibition of prolactin relcase (Lloyd et al., 1975; MacLeod and Lehmeyer, 1974). Transplantation of the pituitary away from dopaminergic influence causes an increase in the secretion of prolactin. Activation of the GABA input to dopamine neurons in the arcuate nucleus may result in an increase in the release of pituitary prolactin. Dopaminergic neurons of the dorsal hypothalamus-zona incerta region may project to the preoptic area, and it has been suggested that they play a role in the modulation of gonadotropin release. Again, GABA synapses on these dopamine neurons would inhibit dopamine release, thereby counteracting the influence of dopamine here. Given the widespread occurrence of GABA throughout the medial hypothalamus and its probable involvement in many hypothalamic functions, it is not surprising that attempts to study the role of GABA in endocrine regulation by the hypothalamus, either by administration of GABA or measurement of hypothalamic GABA after experimental manipulation, have produced complex and sometimes contradictory results.

To conclude, dopaminergic neurons and terminals are found throughout the medial hypothalamus and may be involved in a wide variety of hypothalamic functions. The present experiment demonstrates that GAD immunoreactive axons innervate TH immunoreactive neurons, indicating that the inhibitory amino acid transmitter, GABA, plays a widespread role in the modulation of hypothalamic dopamine neuron activity.

\section{References}

Benisek, W. F., and F. M. Richards (1968) Attachment of metalchelating functional groups to hen egg white lysozyme. J. Biol. Chem. 243: 4267-4271.

Bjorklund, A., O. Lindvall, and A. Nobin (1975) Evidence of an incerto-hypothalamic dopamine neurone system in the rat. Brain Res. 89: $29-42$.

Brown, G. M., E. Krigstein, J. Dankova, and O. Hornykiewica (1972) Relationship between hypothalamic and median eminence catecholamines and thyroid function. Neuroendocrinology 10: 207-217.

Brunk, U., A. Brun, and G. Skold (1966) Histochemical demonstration of heavy metals with the sulphide-silver method. A methodological study. Acta Histochem. 31: 345-357.

Chan-Palay, V., L. Zaborszky, C. Kohler, M. Goldstein, and S. L. Palay (1984) Distribution of tyrosine hydroxylase immunoreactive neurons in the hypothalamus of rats. J. Comp. Neurol. 227: 467-496.

Danscher, G. (1981) Histochemical demonstration of heavy metals. A revised version of the sulphide silver method suitable for both light and electronmicroscopy. Histochemistry $71: 1-16$.

DeMcy, J. (1983) The preparation of immunoglobulin gold conjugates (IGS reagents) and their use as markers for light and electron microscopy. In Immunohistochemistry, A. C. Cuello, ed., pp. 347-372, Wiley, New York.

DeMey, J., M. Moeremans, G. Geuens, R. Nuydens, and M. DeBrabander (1981) High resolution light and electron microscopic localization of tubulin with the IGS (immuno gold staining) method. Cell Biol. Int. Rep. 5: 889-899.

Elias, A. N., L. J. Valenta, A. V. Szekeres, and M. K. Grossman (1982) Regulatory role of gamma-aminobutyric acid in pituitary hormone secretion. Psychoneuroendocrinology 7: 15-30.

Enjalbert, A., M. Ruberg, S. Arancibia, L. Fiore, M. Priam, and C. Kordon (1979) Independent inhibition of prolactin secretion by dopamine and gamma aminobutyric acid in vitro. Endocrinology 105: 823-826.

Everitt, B. J., T. Hökfelt, J. Y. Wu, and M. Goldstein (1984) Coexis-

\footnotetext{
Figure 10. Silver-intensified HRP reaction product: light microscopy. Five sections from the same substantia nigra block were stained with $T H$ antisera simultaneously with peroxidase $(H R P)$ using the ABC method of Hsu et al. (1981). Sections were then intensified with the silver solution $(S I-H R P)$ for $5 \mathrm{~min}(B), 10 \mathrm{~min}(C), 15 \mathrm{~min}(D)$, and $25 \mathrm{~min}(E)$. The section in $A$ was not treated with silver. An increase in the overall density, the number of particles, and the size of particles can be seen. Arrows indicate cells $(B, C)$ and a dendrite $(D)$ with obvious granular depositions. Bar, $20 \mu \mathrm{m}$. F, Ultrastructural view of silver-intensified peroxidasc. Silver particles are dispersed through the cytoplasm of TH immunoreactive profiles from the arcuate nucleus stained with HRP and DAB and intensified with silver nitrate. Outside the HRP immunoreactive profiles, few silver grains are seen. $S I-H R P$, Silver-intensified HRP reaction product. $D$, Dendrite, $A X$, axon. Bar, 275 nm. Colloidal gold was not used for any of the micrographs in Figure 10.
} 
tence of tyrosine hydroxylase-like and gamma-aminobutyric acid-like immunoreactivities in neuron of the arcuate nucleus. Neuroendocrinology 39: 189-191.

Faulk, W. P., and G. M. Taylor (1971) An immunocolloid method for the electron microscope. Immunochemistry $8:$ 1081-1083.

Frens, G. (1973) Controlled nucleation for the regulation of the particle size in monodisperse gold solutions. Nature 241: 20-22.

Fuxe, K. (1964) Cellular localization of monoamines in the median eminence and infundibular stem of some mammals. Z. Zellforsch. Mikrosk. Anat. 61: 710-724.

Geuze, H. J., J. W. Slot, P. A. van der Ley, and R. C. T. Scheffer (1981) Use of colloidal gold particles in double-labelling immunoelectron microscopy of ultrathin frozen tissue sections. J. Cell Biol. 89: 653665 .

Gorcs, T. J., M. Antal, E. Olah, and G. Szekely (1979) An improved cobalt labeling technique with complex compounds. Acta Biol. Acad. Sci. Hung. 30: 79-86.

Haug, F. M. (1967) Electron microscopical localization of the zinc in hippocampal mossy fibre synapses by a modified sulfide procedure. Histochemie 8: 355-368.

Haug, F. M. (1973) A light microscopic study of the rat with Timm's sulphide silver method. Methodological considerations and cytological and regional staining patterns. Adv. Anat. Embryol. Cell Biol. 47: $1-71$

Hodgson, A. J., B. Penke, A. Erdei, I. W. Chubb, and P. Somogyi (1985) Antisera to gamma amino butyric acid: I: Production and characterization using a new model system. J. Histochem. Cytochem. 33: 229239.

Hökfelt, T., K. Fuxe, M. Goldstein, and O. Johansson (1974) Immunohistochemical evidence for the existence of adrenaline neurons in the rat brain. Brain Res. 66: 235-251.

Holgate, C. S., P. Jackson, P. N. Cowen, and C. C. Bird (1983) Immunogold-silver staining: new method of immunostaining with enhanced sensitivity. J. Histochem. Cytochem. 31: 938-944.

I Iorisberger, M. (1979) Evaluation of colloidal gold as a cytochemical marker for transmission and scanning electron microscopy. Biol. Cell. 36: 253-258.

Horisberger, M., and J. Rosset (1977) Colloidal gold, a useful marker for transmission and scanning electron microscopy. J. Histochem. Cytochem. 25: 295-305.

Horisberger, M., D. R. Farr, and M. Vonlanthen (1978) Ultrastructural localization of D-galactan in the nuclei of the myxomycete Physarum polycephalum. Biochem. Biophys. Acta 542: 308-314.

Hsu, S., L. Raine, and H. Fanger (1981) Use of avidin-biotin-peroxidase complex $(\mathrm{ABC})$ in immunoperoxidase techniques: a comparison between $A B C$ and unlabeled antibody (PAP) procedures. J. Histochem. Cytochem. 29: 577-580.

Lloyd, H. M., J. D. Meares, and J. Jacobi (1975) Effects of oestrogen and bromocryptinc on secretion and mitosis in prolactin cell. Nature 255: 497-498.

Lookingland, K. J., and K. E. Moore (1984) Effects of estradiol and prolactin on incertohypothalamic dopaminergic neurons in the male rat. Brain Res. 323: 83-91.

MacLeod, R. M., and J. E. Lehmeyer (1974) Studies on the mechanisms of the dopamine-mediated inhibition of prolactin secretion Endocrinology 94: 1077-1085.

Oertel, W. H., D. E. Schmechel, M. L. Tappaz, and I. Kopin (1981a) Production of a specific antiserum to rat brain glutamic acid decarboxylase by injection of an antigen-antibody complex. Neuroscience 6: 2689-2700.

Oertel, W. H., D. E. Schmechel, E. Mugnaini, M. L. Tappaz, and I. J. Kopin (1981b) Immunocytochemical localization of glutamate decarboxylase in rat cerebellum with a new antiserum. Neuroscience 6: $2715-2735$

Ondo, J. G., and K. A. Pass (1976) The effects of neurally active amino acids on prolactin secretion. Endocrinology 98: 1248-1252.

Pearson, A. A., and S. L. O'Neill (1958) A silver gelatine method for staining nerve fibers. Anat. Rec. 95: 297-301.

Pihl, E. (1967) Ultrastructural localization of heavy metals by a modified sulfide silver method. Histochemie 10: 126-139.

Racagni, G., J. A. Apud, D. Cocchi, V. Locatelli, and E. E. Muller (1982) Gabaergic control of anterior pituitary hormone secretion. Life Sci. 31: 823-838.

Romano, E. L., C. Stolinsky, and C. Hugh-Jones (1974) An antiglobulin reagent labelled with colloidal gold for use in electron microscopy. Immunochemistry $11: 521-522$.
Roth, J. (1982) The preparation of protein A-gold complexes with 3 $\mathrm{nm}$ and $15 \mathrm{~nm}$ gold particles and their use in labelling multiple antigens on ultrathin sections. Histochem. J. 14: 791-801.

Roth, J., M. Bendayan, and L. Orci (1978) Ultrastructural localization of intracellular antigens by the use of protein A-gold complex. J. Histochem. Cytochem. 26: 1074-1081.

Schally, A. V., T. W. Redding, A. Arimura, A. Dupont, and G. L. Linthicum (1977) Isolation of gamma amino butyric acid from pig hypothalami and demonstration of its prolactin release-inhibiting (PIF) activity in vivo and in vitro. Endocrinology 100: 681-691.

Shall, S., and E. A. Barnard (1969) Heavy atom-labelled derivatives of bovine pancreatic ribonuclease. I. Specific reactions of ribonuclease with $\mathrm{N}$-acetylhomocysteine thiolactone and silver ion. J. Mol. Biol. 41: 237-251.

Slot, J. W. (1984) Handout on methods in immunogold labeling of cryosections. In Cryoultramicrotomy and Immunolabeling of Ultrathin Sections, K. Fisher and L. Furcht, eds., American Society for Cell Biology.

Slot, J. W., and H. J. Geuze (1983) The use of protein A-colloidal gold (PAG) complexes as immunolabels in ultra-thin frozen sections. In Immunohistochemistry, A. C. Cuello, ed., pp. 323-346, Wiley, New York.

Somogyi, P., A. J. Hodgson, I. A. Chubb, B. Penke, and A. Erdei (1985) Antiserum to gamma-butyric acid: II. Immunocytochemical application to the central nervous system. J. Histochem. Cytochem. 33: 240-248.

Stathis, E. C., and A. Fabrikanos (1958) Preparation of colloidal gold. Chem. Ind. 27: 860-861.

Sternberger, L. A. (1979) Immunocytochemistry, Wiley, New York.

Sternberger, L., J. S. Hanker, E. J. Donati, J. P. Petrali, and A. M. Seligman (1966) Method for enhancement of electron microscopic visualization of embedded antigen by bridging osmium to uranium antibody with thiocarbohydrazide. J. Histochem. Cytochem. 14: 711718.

Swanson, L. W., and B. K. Hartman (1975) The central adrencrgic system. An immunofluorescence study of the location of cell bodies and their efferent connections in the rat utilizing dopamine-beta hydroxylase as a marker. J. Comp. Neurol. 163: 467-506.

Swanson, L. W., P. E. Sawchenko, A. Berod, B. K. Hartman, K. B. Helle, and D. E. Vanorden (1981) An immunohistochemical study of the organization of catecholaminergic cells and terminal fields of the paraventricular and supraoptic nuclei of the hypothalamus. $J$. Comp. Neurol. 196: 271-285.

Szentagothai, J., B. Flerko, B. Mess, and B. Halasz (1972) Hypothalamic Control of the Anterior Pituitary. Akademiai Kiado, Budapest.

Tappaz, M. L. (1984) GABA and anterior pituitary function: anatomical data. Psychoneuroendocrinology 9: 85-95.

Tappaz, M. L., W. H. Oertel, M. Wassef, and E. Mugnaini (1982) Central GABAergic neuroendocrine regulations: pharmacological and morphological evidence. Prog. Brain Res. 55: 77-96.

Tappaz, M. L., M. Wassef, W. H. Oertel, L. Paut, and J. F. Pujol (1983) Light- and electron-microscopic immunocytochemistry of glutamic acid decarboxylase (GAD) in the basal hypothalamus: morphological evidence for neuroendocrine gamma-aminobutyrate (GABA). Neuroscience 9: 271-287.

Tilders, F. J. H., H. A. Van der Woude, D. F. Swaab, and A. H. Mulder (1979) Identification of MSH release-inhibiting elements in the neurointermediate lobe of the rat. Brain Res. 171: 425-435.

Tyrer, N. M., and E. M. Bell (1974) The intensification of cobalt-filled neurone profiles using a modification of Timm's sulphide silver method. Brain Res. 73: 151-155.

van den Pol, A. N. (1983) Colloidal gold and peroxidase as ultrastructural markers for simultaneous localization of two neurotransmitter antigens in the hypothalamic paraventricular nucleus. Soc. Neurosci. Abstr. 9: 84

van den Pol, A. N. (1984a) Colloidal gold and biotin-avidin conjugates as ultrastructural markers for neural antigens. Q. J. Exp. Physiol. 69: $1-33$.

van den Pol, A. N. (1984b) Double pre-embedding ultrastructural immunocytochemistry with intensified colloidal gold and peroxidase: Dopamine neurons in the dorsomedial hypothalamus receive GABA synapses. Soc. Neurosci. Abstr. 10:440.

van den Pol, A. N. (1985a) Silver intensified gold and peroxidase as simultaneous ultrastnuctural markers for pre- and postsynaptic neurotransmitters. Science 228: 332-335.

van den Pol, A. N. (1985b) Dual ultrastructural localization of two 
neurotransmitter-related antigens: Colloidal gold labeled neurophysin immunoreactive supraoptic neurons receive peroxidase labeled glutamate decarboxylase or gold labeled GABA immunoreactive synapses. J. Neurosci. 5: 2940-2954.

van den Pol, A. N., and J. R. Cassidy (1982) The hypothalamic arcuate nucleus of rat-a quantitative Golgi analysis. J. Comp. Neurol. 204: 65-98.

van den Pol, A. N., and K. Tsujimoto (1985) Neurotransmitters of the hypothalamic suprachiasmatic nucleus-immunocytochemical analysis of 22 neuronal antigens. Neuroscience 15: 1049-1086.

van den Pol, A. N., R. Herbst, and J. F. Powell (1984) Tyrosine hydroxylase immunoreactive neurons of the hypothalamus: a light and electron microscopic study. Neuroscience 13: 1117-1154.

Vincent, S. R., T. Hökfelt, and J. Y. Wu (1982) GABA neuron systems in hypothalamus and the pituitary gland. Neuroendocrinology 34 : 117-125.

Weiner, R. I., and W. F. Ganong (1978) Role of brain monoamines and histamine in regulation of anterior pituitary secretion. Physiol. Rev. 58: 905-976.

Wu, J.-Y. (1983) Preparation of glutamic acid decarboxylase as immunogen for immunocytochemistry. In Immunohistochemistry, C. Cuello, ed., pp. 159-191, Wiley, New York. 